University of New Hampshire

University of New Hampshire Scholars' Repository

Space Science Center

Institute for the Study of Earth, Oceans, and

Space (EOS)

2006

\title{
Radiation Damage and Activation from Proton Irradiation of Advanced Scintillators
}

\author{
Peter F. Bloser \\ University of New Hampshire, Peter.Bloser@unh.edu \\ Mark L. McConnell \\ University of New Hampshire - Main Campus, mark.mcconnell@unh.edu \\ John R. Macri \\ University of New Hampshire - Main Campus, John.Macri@unh.edu \\ P J. Bruillard \\ University of New Hampshire - Main Campus \\ James M. Ryan \\ University of New Hampshire, James.Ryan@unh.edu
}

See next page for additional authors

Follow this and additional works at: https://scholars.unh.edu/ssc

Part of the Astrophysics and Astronomy Commons

\section{Recommended Citation}

Bloser, P.F.; McConnell, M.L.; Macri, J.R.; Bruillard, P.J.; Ryan, J.M.; Hajdas, W., "Radiation Damage and Activation from Proton Irradiation of Advanced Scintillators," Nuclear Science Symposium Conference Record, 2006. IEEE , vol.3, no., pp.1500,1505, Oct. 29 2006-Nov. 12006

This Conference Proceeding is brought to you for free and open access by the Institute for the Study of Earth, Oceans, and Space (EOS) at University of New Hampshire Scholars' Repository. It has been accepted for inclusion in Space Science Center by an authorized administrator of University of New Hampshire Scholars' Repository. For more information, please contact Scholarly.Communication@unh.edu. 


\section{Authors}

Peter F. Bloser, Mark L. McConnell, John R. Macri, P J. Bruillard, James M. Ryan, and W Hajdas 


\title{
Radiation Damage and Activation from Proton Irradiation of Advanced Scintillators
}

\author{
Peter F. Bloser, Mark L. McConnell, John R. Macri, Paul J. Bruillard, James M. Ryan, Wojtek Hajdas
}

\begin{abstract}
We present results from a proton accelerator beam test to measure radiation damage and activation in advanced scintillator materials. Samples of $\mathrm{LaBr}_{3}: \mathrm{Ce}$ and $\mathrm{LaCl}_{3}: \mathrm{Ce}$ were exposed to protons from 40-250 $\mathrm{MeV}$ at the Proton Irradiation Facility of the Paul Scherrer Institute in Switzerland. Twelve energy bands were used to simulate the spectrum of the South Atlantic Anomaly (SAA), with different samples exposed to the equivalent of 4 months, 1 year, and 5 years of SAA passage. No significant decrease in light output was found due to radiation damage, indicating that these new scintillator materials are radiation tolerant. High-resolution spectra of the samples were obtained before and after irradiation with a Germanium spectrometer to study activation. We present a detailed analysis of these spectra and a discussion of the suitability of these scintillator materials for detectors in future space missions.
\end{abstract}

\section{INTRODUCTION}

Newly-developed advanced scintillator materials, such as the cerium-doped lanthanum halides $\left(\mathrm{LaBr}_{3}: \mathrm{Ce}, \mathrm{LaCl}_{3}: \mathrm{Ce}\right)$, show great promise for gamma-ray detectors in future space-based astrophysics missions [1]-[3]. Their high density, high atomic number, high light output, fast decay times, and good linearity provide excellent stopping power, energy resolution, and timing properties [4]. Such detectors are potentially competitive in spectroscopy performance with solid-state detectors such as CZT, with the considerable advantages of lower material cost and well-understood, simplified readout using standard photomultiplier tube (PMT) technology.

High-energy astrophysics missions must be carried out in space, where detectors are subjected to intense photon and particle background radiation. It is thus important to verify that a given detector material is not overly sensitive to radiation damage, and that any activation of the material does not produce unacceptable levels of background. One method of checking this is to expose samples of the detector material to radiation from particle accelerators in doses equivalent to what is expected in space. In this paper we report on irradiation tests designed to mimic the proton exposure expected for an instrument in a low Earth orbit (LEO). The primary particle background in this environment is due to trapped protons in the Earth's radiation belts, particularly in the South Atlantic Anomaly (SAA).

Manuscript received November 17, 2006. This work was supported by the NASA APRA program.

P. F. Bloser, M. L. McConnell, J. R. Macri, P. J. Bruillard, and J. M. Ryan are with the Space Science Center, University of New Hampshire, Durham, NH 03824, USA (telephone: 603-862-0289, e-mail: Peter.Bloser@unh.edu).

W. Hajdas is with the Paul Scherrer Institute, CH-5232 Villigen, Switzerland

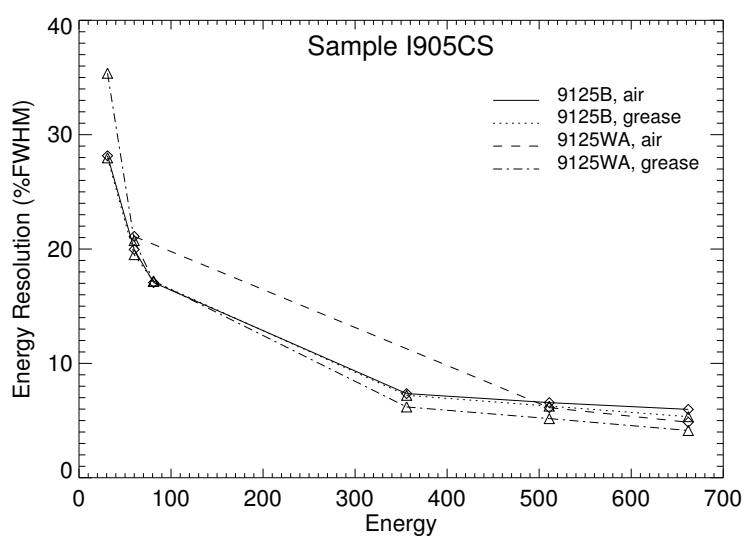

Fig. 1. Energy resolution as a function of energy for a Saint-Gobain sample of $\mathrm{LaBr}_{3}$. The resolution depends slightly on the optical coupling and on the type of glass in the PMT.

\section{SCINTILLATOR SAMPLES}

We acquired small $(0.5 " \times 0.5 "$ cylinder $)$ samples of scintillator material for exposure to proton irradiation. These included six samples of BrilLanCe $\mathrm{TM}_{380}\left(\mathrm{LaBr}_{3}: \mathrm{Ce}\right)$ and six samples of BrilLanCe ${ }^{\mathrm{TM}} 350\left(\mathrm{LaCl}_{3}: \mathrm{Ce}\right)$ from Saint-Gobain Crystals $(5 \%$ Ce concentration). The samples were hermetically sealed in aluminum a quartz optical window. They did not include an internal optical coupling between the crystal and optical window in order to reduce the amount of passive material exposed to the proton irradiation. In addition, Radiation Monitoring Devices (RMD), Inc. provided additional samples of $\mathrm{LaBr}_{3}$ and $\mathrm{LaCl}_{3}$ material in the form of small chips immersed in oil and contained in small plastic vials.

The Saint-Gobain samples were characterized in the laboratory using radioactive gamma-ray line sources. The energy resolution was measured as a function of energy using two different photomultiplier tubes (PMTs) with both an air coupling and an optical grease coupling (Fig. 1). The comparison of the two PMTs was motivated by the fact that the energy resolution quoted in the Saint-Gobain data sheet that accompanied the samples was obtained using a PMT with UV-transmitting glass (the Hamamatsu R4017). We found that using a UV-sensitive PMT (Electron Tubes 9125WA) resulted in slightly better energy resolution than using a PMT with standard borosilicate glass (9125B): 4.1\% FWHM vs. 5.3\% FWHM at $662 \mathrm{keV}$. (We note that the energy resolution obtained with these samples is slightly worse than that normally quoted in the literature for 


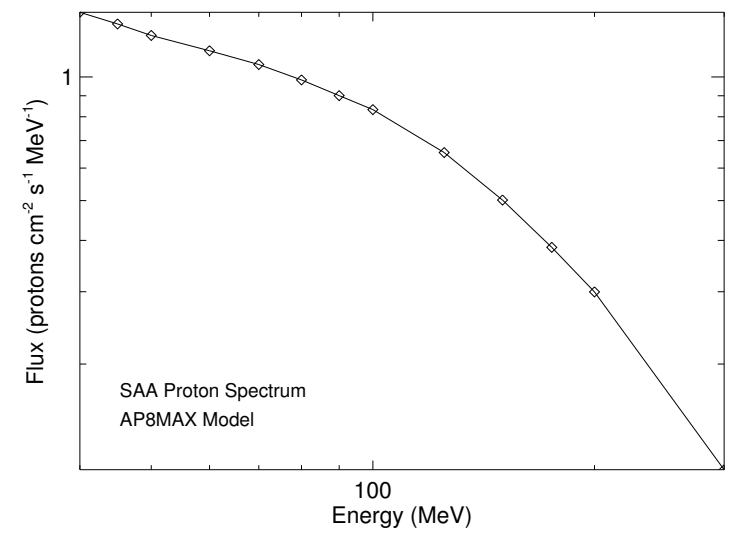

Fig. 2. Average differential proton flux spectrum in the SAA calculated using the AP8MAX model and the SPENVIS package.

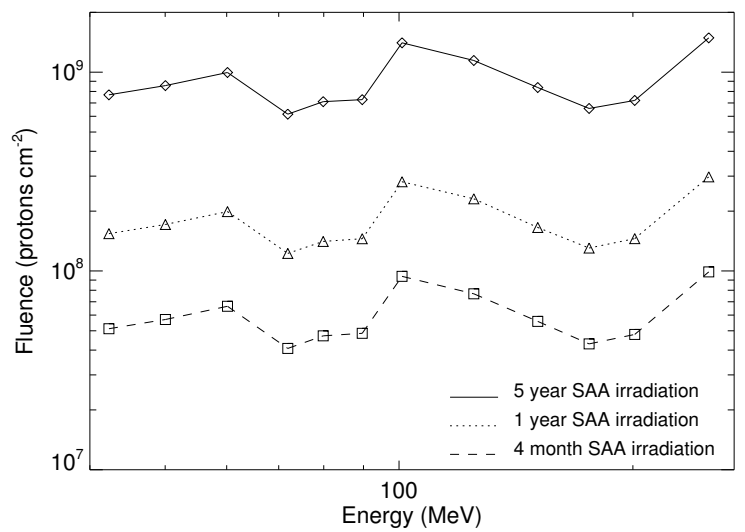

Fig. 3. Proton fluence spectra generated at PIF. The samples were exposed in three batches to represent the total fluence expected from the SAA after 4 months, 1 year, and 5 years in orbit. Note that the bin size increases at higher energies.

$\mathrm{LaBr}_{3}, \sim 2.7 \%$ at $662 \mathrm{keV}$, due to the lack of an internal optical coupling.) We are not certain why this should be, as $\mathrm{LaBr}_{3}$ has not been reported to have significant scintillation light emission at UV wavelengths.

\section{PRoton IRRAdiation TEST}

We exposed the scintillator samples to proton irradiation at the Proton Irradiation Facility (PIF) at the Paul Scherrer Institute (PSI) in Villigen, Switzerland on 18 December 2005. Protons were produced in 12 energy bands between $40 \mathrm{MeV}$ and $250 \mathrm{MeV}$ to simulate the average SAA spectrum, which was calculate using the AP8MAX model and the SPENVIS package from ESA [5] (Fig. 2). The total fluence in each energy band is shown in Fig. 3; note that the bin size increases at higher energy, making the spectrum look flatter than the differential flux spectrum in Fig. 2. The samples were exposed in three batches to represent the total SAA exposure after 4 months, 1 year, and 5 years in orbit. The total $(40-250 \mathrm{MeV})$ fluence was $7.3 \times 10^{8}$ protons $\mathrm{cm}^{-2}$ for the 4-month exposure, $2.2 \times 10^{9}$

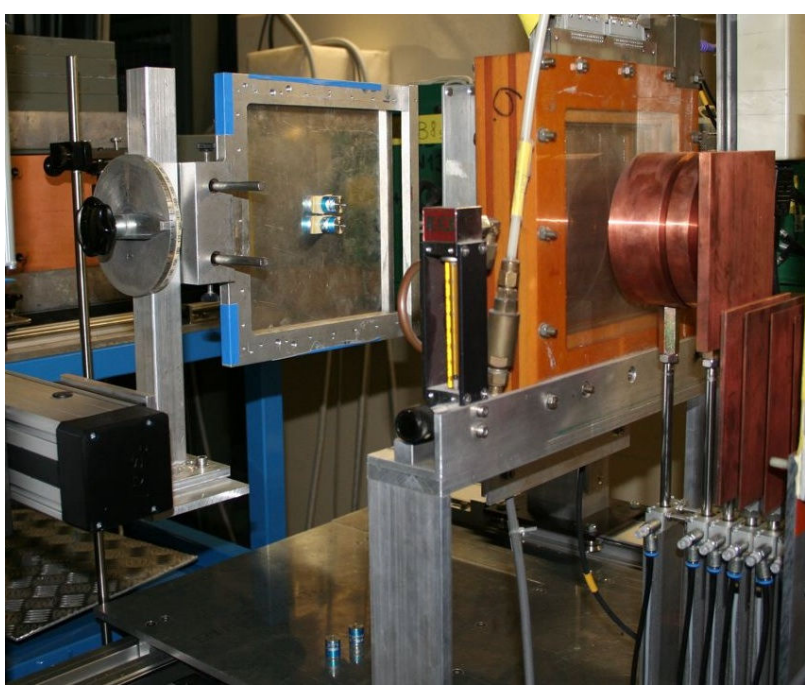

Fig. 4. Sample frame at PIF, showing irradiated samples and control samples (at bottom).

protons $\mathrm{cm}^{-2}$ for the 1-year exposure, and $1.1 \times 10^{10}$ protons $\mathrm{cm}^{-2}$ for the 5-year exposure.

Each batch of Saint-Gobain samples comprised one $\mathrm{LaBr}_{3}$ and one $\mathrm{LaCl}_{3}$ crystal in the proton beam ( $\sim 8 \mathrm{~cm}$ beam size) and one of each crystal placed nearby but out of the beam (the "controls"). The RMD samples were exposed in separate batches, but there were not enough of these available to use controls. The beam sample frame, with Saint-Gobain irradiated samples and controls, is shown in Fig. 4.

After the irradiation, two sets of measurements were made for the irradiated samples and the controls, both repeated several times over the following 24 hours: to study possible radiation damage and internal background due to activation, spectra from a ${ }^{22} \mathrm{Na}$ source were recorded for each detector using a PMT and multi-channel analyzer. To study the gammaray lines produced by the proton activation, the samples were placed in front of a calibrated high-purity Ge spectrometer.

\section{RESULTS}

\section{A. Radiation Damage}

We looked for radiation damage by comparing the photoelectron (p.e.) yield of each sample before and after irradiation [6]. We recorded a ${ }^{22} \mathrm{Na}$ spectrum in each of the samples, which were placed on the entrance window of a 1.5" PMT. The before and after spectra of the 5-year-irradiated $\mathrm{LaBr}_{3}$ sample are shown in Figs. 5(a) and 5(b), respectively. The two lines (511 keV and $1275 \mathrm{keV}$ ) of ${ }^{22} \mathrm{Na}$ were used to calibrate the spectrum and subtract any offset, and the centroid $(C)$ and width $(\sigma)$ of the $511 \mathrm{keV}$ photopeak was calculated. Then the p.e. yield (p.e. $\mathrm{keV}^{-1}$ ) is given, to within a factor of order unity, by $(C / \sigma)^{2} / 511$.

The calculated p.e. yield of the 5-year-irradiated SaintGobain $\mathrm{LaBr}_{3}$ sample is shown in Fig. 6, along with its control sample. There is evidence of slightly reduced yield following the irradiation. In Fig. 7, however, we show the p.e. yield of 


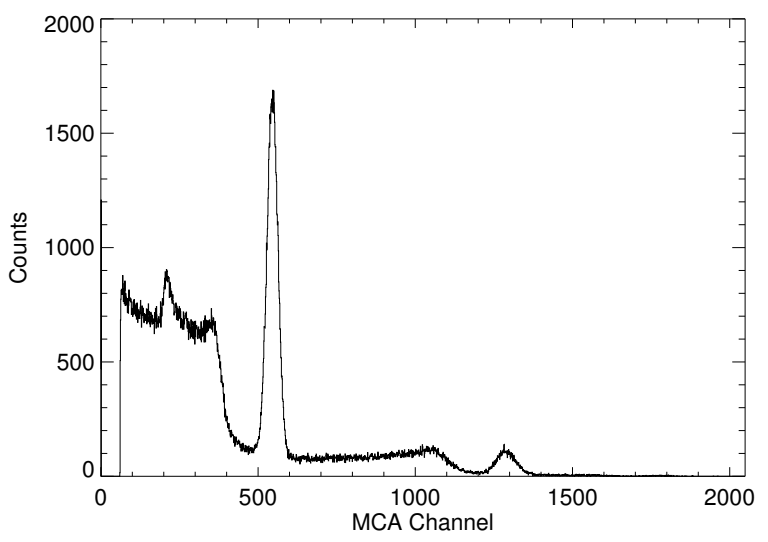

(a) Before irradiation.

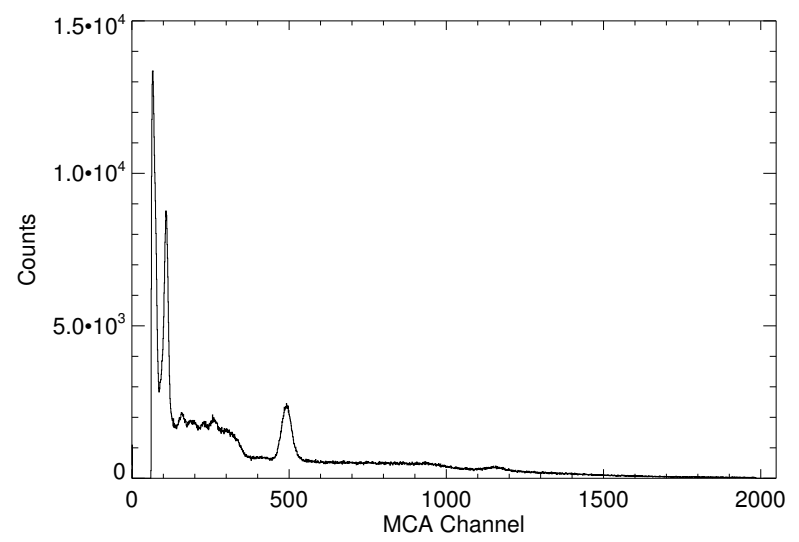

(b) After irradiation.

Fig. 5. ${ }^{22} \mathrm{Na}$ spectra recorded in a $\mathrm{LaBr}_{3}$ sample before (a) and after (b) exposure to the equivalent proton fluence of 5 years of SAA passages. The bright lines at low energy in (b) are due to activation. The $511 \mathrm{keV}$ and $1275 \mathrm{keV}$ photopeaks from ${ }^{22} \mathrm{Na}$ are visible in both spectra.

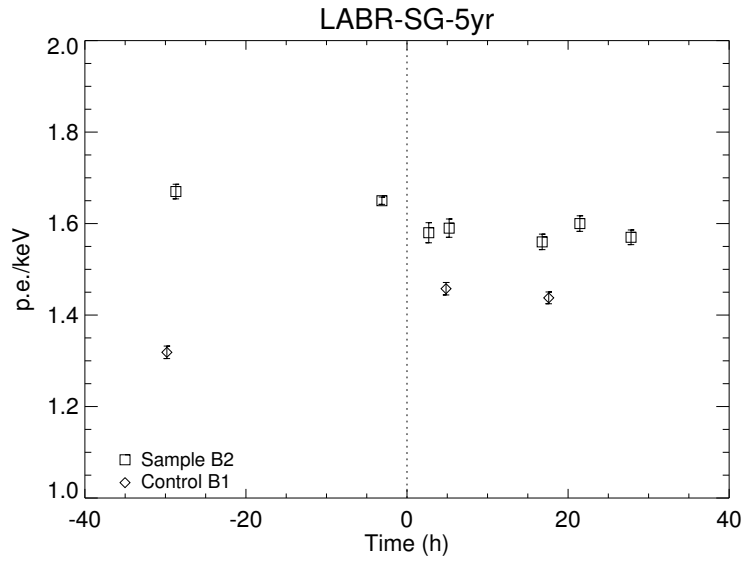

Fig. 6. Photoelectron yield of Saint-Gobain $\mathrm{LaBr}_{3}$ sample exposed to 5-year SAA irradiation. Control sample is also plotted.

the $\mathrm{RMD} \mathrm{LaBr}_{3}$ samples, which are contained in a different packaging. These do not show any evidence of reduced p.e. yield. In both figures the error bars are purely statistical. We have found that there is an additional $\sim 3 \%$ systematic variation in the p.e. yield from a given sample when it is removed from and replaced on the PMT several times in succession. The apparent increase in sample D2 in Fig. 7 is likely due to that sample's irregular shape, which makes it especially difficult to reliably record the same light yield in successive measurements. Other samples do not show a clear trend in p.e. yield with time, before or after the irradiation. We believe that the decrease in p.e. yield in Fig. 6 is likely due to a darkening of the quartz window and not to damage to the crystal. We conclude that there is no evidence of significant radiation damage in $\mathrm{LaBr}_{3}$ or $\mathrm{LaCl}_{3}$ due to proton irradiation.

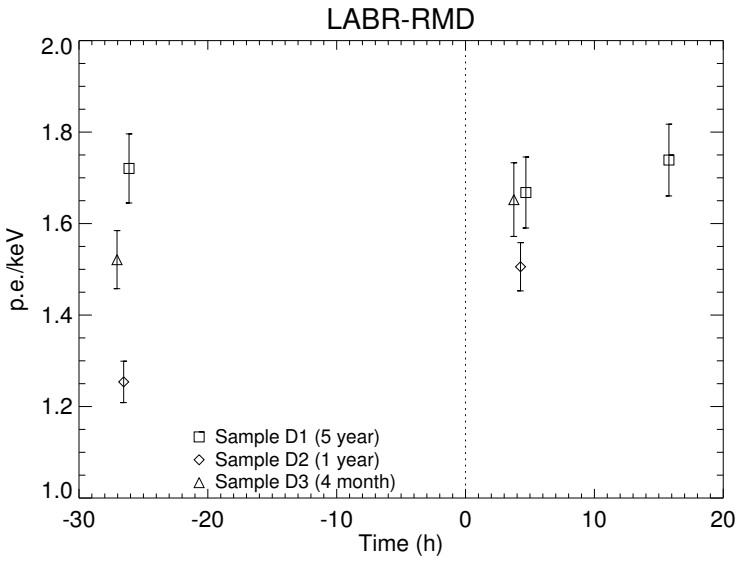

Fig. 7. Photoelectron yield of all three $\mathrm{RMD} \mathrm{LaBr}_{3}$ samples.

\section{B. Activation Lines}

In Fig. 8 we show the Ge spectrum of the 5-year-irradiated Saint-Gobain $\mathrm{LaBr}_{3}$ sample obtained 1.2 hours after irradiation. The fitting and identification of the lines was performed at PSI. Lines observed include ${ }^{130} \mathrm{La},{ }^{131} \mathrm{La},{ }^{132} \mathrm{La},{ }^{132} \mathrm{Ce},{ }^{135} \mathrm{Ce},{ }^{75} \mathrm{Br}$, ${ }^{77} \mathrm{Br},{ }^{77} \mathrm{Kr}$, ${ }^{73} \mathrm{Se}$ and ${ }^{76} \mathrm{As}$ (and ${ }^{54} \mathrm{Mn}$ and ${ }^{24} \mathrm{Na}$ from the $\mathrm{Al}$ housing). These are all fairly short-lived species, with half-lives of 1-2 days or less.

In Fig. 9 we show the Ge spectrum of the 5-year-irradiated Saint-Gobain $\mathrm{LaCl}_{3}$ sample obtained 1.3 hours after irradiation. The observed lines include ${ }^{131} \mathrm{La},{ }^{132} \mathrm{La},{ }^{132} \mathrm{Ce},{ }^{133} \mathrm{Ce},{ }^{135} \mathrm{Ce}$, ${ }^{127} \mathrm{Cs}$ and ${ }^{129} \mathrm{Cs}$ (and ${ }^{24} \mathrm{Na}$ from $\mathrm{Al}$ housing). The $\mathrm{LaCl}_{3}$ shows essentially the same lines, minus the $\mathrm{Br}$ species, as expected. The total count rate is lower than for the $\mathrm{LaBr}_{3}$ sample. 


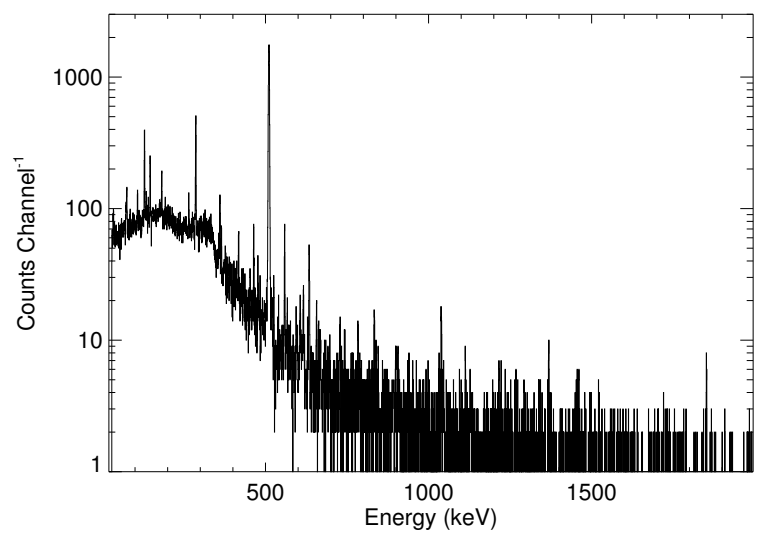

Fig. 8. Ge spectrum of activation lines from Saint-Gobain $\operatorname{LaBr}_{3}, 1.2$ hours after irradiation with 5-year SAA proton fluence.

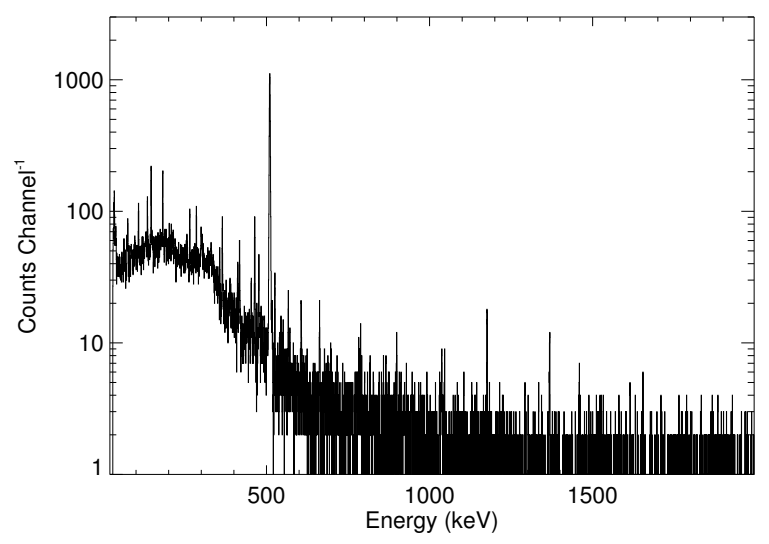

Fig. 9. Ge spectrum of activation lines from Saint-Gobain $\mathrm{LaCl}_{3}, 1.3$ hours after irradiation with 5-year SAA proton fluence.

\section{Internal Activation Background}

To study the internal background due to activation we subtracted the pre-irradiation ${ }^{22} \mathrm{Na}$ spectrum (e.g., Fig. 5(a)) from the post-irradiation spectra (e.g., Fig. 5(b)) for each sample, after calibrating and rebinning each spectrum in energy. The resulting internal background spectra for the 5-year-irradiated Saint-Gobain $\mathrm{LaBr}_{3}$ sample is shown in Fig. 10. A beta-decay continuum is evident, as well as several lines that decay rapidly. In Fig. 11 we focus on the lines below $600 \mathrm{keV}$. The longestlasting line is around $240 \mathrm{keV}$, probably due mainly to ${ }^{77} \mathrm{Br}$. There is also evidence of ${ }^{77} \mathrm{Kr}$ at $\sim 130 \mathrm{keV}$. There is also a bright line at $\sim 70 \mathrm{keV}$ that decays away with a half life of about 5 hours. The origin of this line is not clear. An important clue is that it also appears in the control sample, albeit much more weakly, and we therefore conclude that it must be caused by neutron activation. Monte Carlo simulations of the proton beam setup predict a flux of $\sim 10^{-4}$ neutrons $\mathrm{cm}^{-2}$ proton $^{-1}$ above $1 \mathrm{MeV}$ at the sample frame, and so the 5-year irradiated samples should have received $\sim 10^{5}$ neutrons $\mathrm{cm}^{-2}$. The only isotope that we have been able to identify at

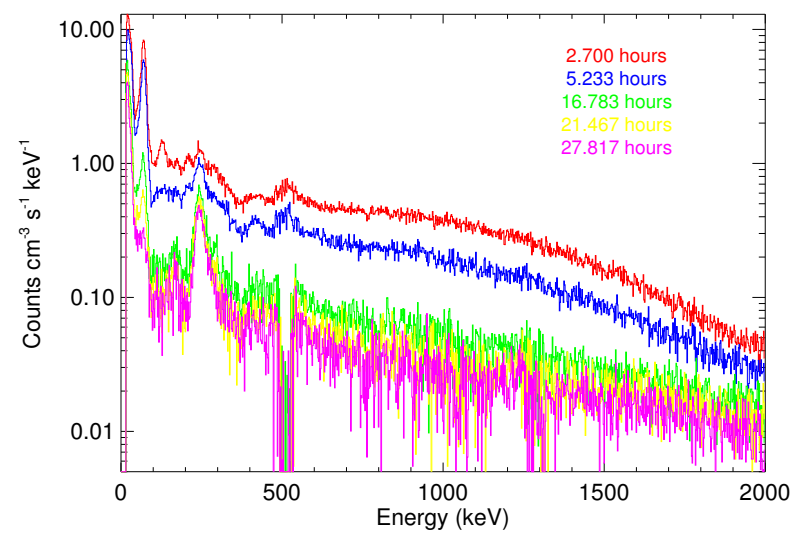

Fig. 10. Internal background due to activation in the 5-year-irradiated SaintGobain $\mathrm{LaBr}_{3}$ sample at various times after irradiation.

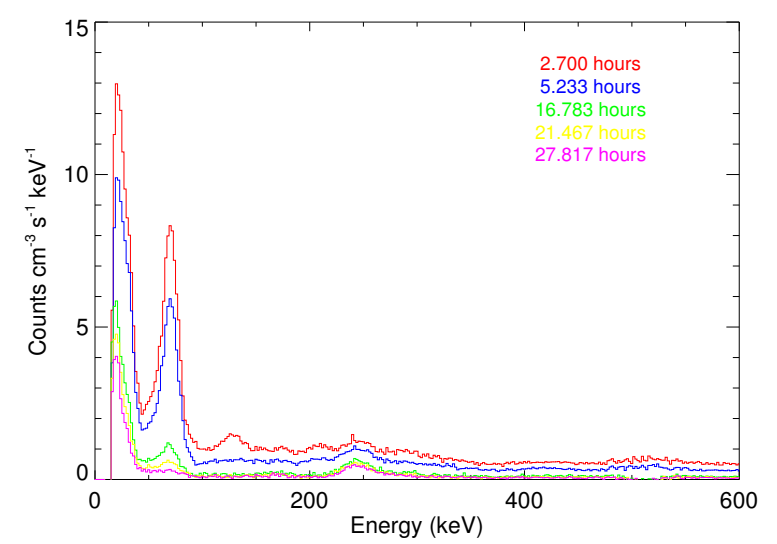

Fig. 11. Internal background lines below $600 \mathrm{keV}$ due to activation in the 5-year-irradiated Saint-Gobain $\mathrm{LaBr}_{3}$ sample.

the time of this writing that fits the data is ${ }^{111 m} \mathrm{Pd}$, which has a half life of 5.5 hours, lines at $70.4 \mathrm{keV}$ and $172.2 \mathrm{keV}$ (there is evidence of both in Fig. 11), and is produced by fast and thermal neutron activation. There is no reason to expect this isotope to be present, however.

The internal background due to activation in the 5-yearirradiated Saint-Gobain $\mathrm{LaCl}_{3}$ sample is shown in Figs. 12 and 13. Continuum and line emission is seen in this sample as well. We note that the energy resolution in this sample is poorer than that in the $\mathrm{LaBr}_{3}$ sample shown in Figs. 10 and 11. The longest-lasting line is at $\sim 250 \mathrm{keV}$, probably due to ${ }^{137 m} \mathrm{Ce}$ (which must contribute to the line near $240 \mathrm{keV}$ in Fig. 11 as well). Notably, there is no line evident at $70 \mathrm{keV}$. This makes the identification of the isotope responsible for the $70 \mathrm{keV}$ line in the $\mathrm{LaBr}_{3}$ samples even more problematic.

The total (15-2000 keV) internal counting rate due to activation is shown for $\mathrm{LaBr}_{3}$ in Fig. 14 and for $\mathrm{LaCl}_{3}$ in Fig. 15. For both $\mathrm{LaBr}_{3}$ and $\mathrm{LaCl}_{3}$ the counting rate decays on a time scale of about 10-15 hours. A satellite in LEO would pass through the SAA on 2-3 consecutive orbits, and then would miss it for most 


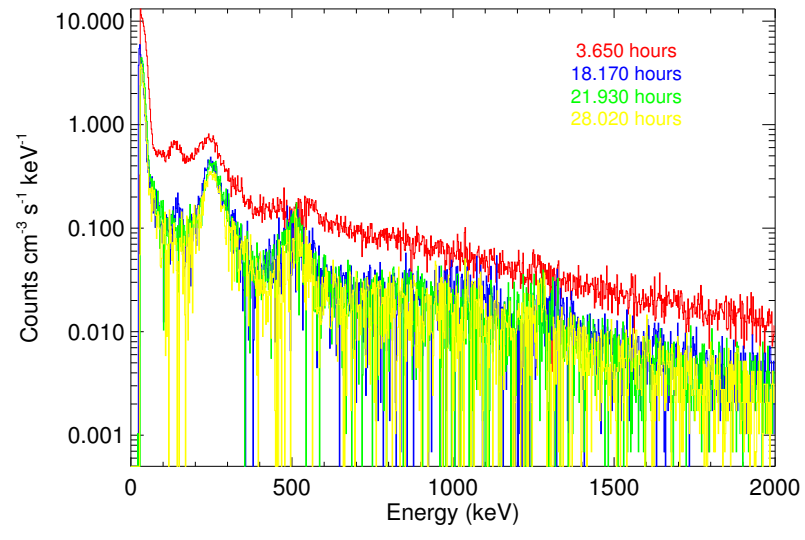

Fig. 12. Internal background due to activation in the 5-year-irradiated SaintGobain $\mathrm{LaCl}_{3}$ sample at various times after irradiation.

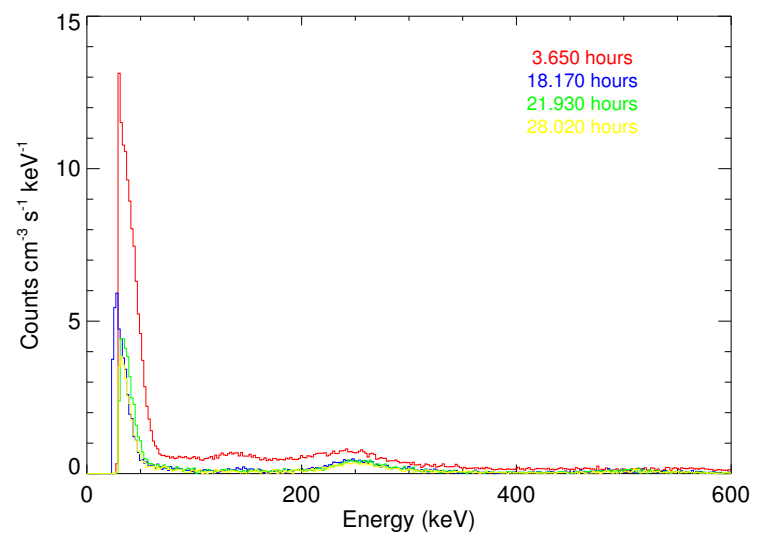

Fig. 13. Internal background lines below $600 \mathrm{keV}$ due to activation in the 5-year-irradiated Saint-Gobain $\mathrm{LaCl}_{3}$ sample.

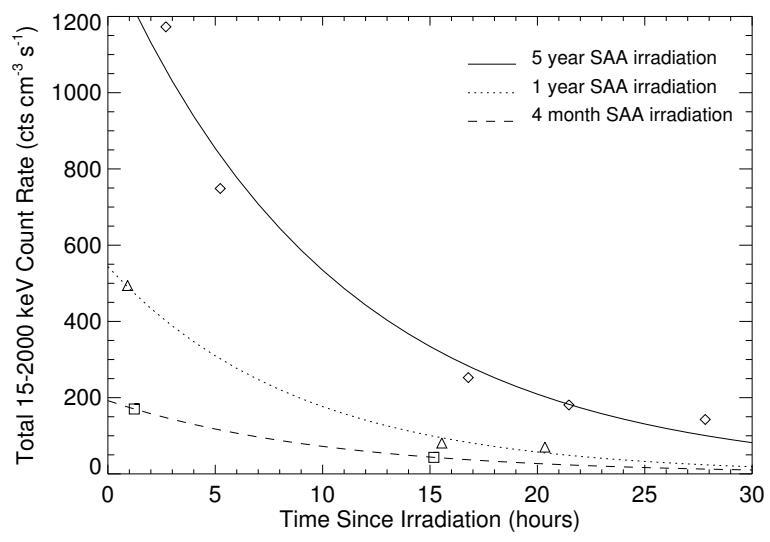

Fig. 14. Total internal counting rate $(15-2000 \mathrm{keV})$ due to activation in $\mathrm{LaBr}_{3}$ for three different proton fluences.

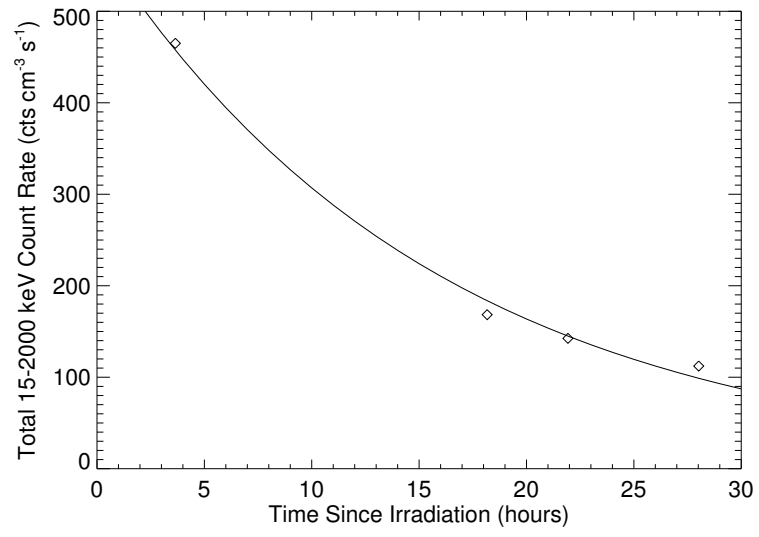

Fig. 15. Total internal counting rate $(15-2000 \mathrm{keV})$ due to activation in $\mathrm{LaCl}_{3}$ for a proton fluence equivalent to 5 years of SAA passages.

of a day. Scaling from Figs. 14 and 15, the internal background due to several hours of SAA exposure would be $<<10$ cts $\mathrm{cm}^{-3} \mathrm{~s}^{-1}$, and this would substantially decay away before the next set of passages. We therefore conclude that proton activation should not be a dominant source of background for an instrument in LEO employing $\mathrm{LaBr}_{3}$ or $\mathrm{LaCl}_{3}$ detectors.

\section{CONCLUSION}

We have found that $\mathrm{LaBr}_{3}$ and $\mathrm{LaCl}_{3}$ do not suffer significant radiation damage after proton irradiation equivalent to 5 years in LEO, and that activation is short-lived and should not be a dominant source of background. We note that similar results have recently been reported elsewhere [7]. Our next task will be to understand the activation in detail using Monte Carlo modeling. Specifically, we plan to use MGGPOD [8], [9], a simulation suite designed to model gamma-ray background in space missions, to reproduce the results of the proton irradiation test. With our simulation tools validated, we will be in a position to confidently predict the sensitivity of specific future gamma-ray astronomy missions based on advanced scintillators.

\section{ACKNOWLEDGMENT}

The authors would like to thank K. Shah of RMD, Inc. for supplying samples of $\mathrm{LaBr}_{3}$ and $\mathrm{LaCl}_{3}$ for the beam test.

\section{REFERENCES}

[1] M. L. McConnell et al., "The CASTER black hole finder probe," New Astronomy Reviews, vol. 50, p. 633, 2006.

[2] — - "CASTER: a concept for a black hole finder probe based on the use of new scintillator technologies," in UV, X-Ray, and Gamma-Ray Space Instrumentation for Astronomy XIV, ser. Proc. SPIE, O. H. W. Siegmund, Ed., vol. 5898, 2005, p. 1.

[3] P. F. Bloser, S. D. Hunter, J. M. Ryan, M. L. McConnell, and J. R. Marcri, "Gas micro-well track imaging detectors for gamma-ray astronomy," in UV, X-Ray, and Gamma-Ray Space Instrumentation for Astronomy XIV, ser. Proc. SPIE, O. H. W. Siegmund, Ed., vol. 5898, 2005, p. 152.

[4] K. S. Shah et al., " $\mathrm{LaBr}_{3}$ :Ce scintillators for gamma ray spectroscopy," IEEE Transactions on Nuclear Science, vol. 50, p. 2410, 2003.

[5] D. Heynderickx. (2004) SPENVIS - space environtment, effects, and education system. [Online]. Available: http://www.spenvis.oma.be/spenvis/ 
[6] R. Georgii et al., "Influence of radiation damage on BGO scintillation properties," Nuclear Instruments and Methods in Physics Research A, vol. 413, p. 50, 1998.

[7] E. Buis et al., "New scintillators for focal plane detectors in gamma-ray missions," Experimental Astronomy, vol. 20, p. 333, 2006.
[8] G. Weidenspointner et al., "MGGPOD: a Monte Carlo suite for modeling instrumental line and continuum backgrounds in gamma-ray astronomy," Astrophysical Journal Supplement Series, vol. 156, pp. 69-91, 2005.

[9] C. B. Wunderer et al., "The ACT vision mission study simulation effort," New Astronomy Reviews, vol. 50, p. 608, 2006. 